08.3

\title{
Энергетический спектр и волновые функции электронов в туннельно-связанных сферических квантовых точках InAs/GaAs
}

\author{
(С Г.Ф. Глинский, Д.А. Шапран \\ Санкт-Петербургский государственный электротехнический университет „ЛЭТИ“, Санкт-Петербург, Россия \\ E-mail: genaglinskii@mail.ru
}

Поступило в Редакцию 7 ноября 2019 г.

В окончательной редакции 7 ноября 2019г.

Принято к публикации 16 декабря 2019г.

\begin{abstract}
Представлены результаты численного расчета энергии основного состояния электрона и электронной плотности в туннельно-связанных квантовых точках InAs/GaAs, образующих простую кубическую сверхрешетку. Расчет проводится в рамках модифицированного метода эффективной массы, учитывающего микроскопическое (атомарное) строение отдельных квантовых точек, без учета спин-орбитального взаимодействия и деформационных эффектов. Исследована зависимость энергии связи электрона от радиуса квантовых точек $R$. Показано, что в области $R<27 \AA$ энергия связи электрона пропорциональна $R^{3}$.
\end{abstract}

Ключевые слова: квантовые точки, сверхрешетки, электронная плотность.

DOI: 10.21883/PJTF.2020.06.49159.18104

Расчет энергетического спектра и волновых функций носителей заряда в квантовых точках (КТ) относится к числу важнейших задач современной наноэлектроники. В настоящее время можно выделить два основных подхода к решению этой задачи. В основе первого из них лежат методы, приближающиеся к $a b$ initio: это метод функционала плотности (DFT) [1] и метод псевдопотенциала [2-6]. Для реализации обоих методов требуются значительные вычислительные ресурсы. Второй подход основан на использовании традиционного метода эффективной массы в его континуальном приближении [7-10]. Однако неоднозначность определения эффективного $k$-гамильтониана гетероструктуры (ГС), a также граничных условий для огибающих волновых функций не позволяет использовать его для расчета КТ малых размеров и сверхрешеток $(\mathrm{CP})$ на их основе.

В работах [11-13] в рамках метода эффективной массы предложен новый подход к определению энергетического спектра и волновых функций носителей заряда в полупроводниковых ГС, таких как квантовые ямы, проволоки, КТ и СР. В основе этого подхода лежит введение характеристической функции $f(\mathbf{a})$, указывающей на замещение в элементарной ячейке с номером а атомов опорного кристалла атомами другого материала. В отличие от обычно используемого континуального приближения данный метод позволяет учесть микроскопическое строение ГС, а значит, ее реальную симметрию, включая симметрию интерфейсов. Было показано, что он может быть реализован в двух унитарно эквивалентных k- и а-представлениях. Для одиночных ГС конечных размеров и СР определение энергетического спектра и волновых функций носителей заряда в обоих представлениях сводится к поиску собственных чисел и собственных столбцов эффективного матричного гамильтониана. В k-представлении такой гамильтониан можно определить, используя $k p$-теорию возмущений или метод инвариантов $[11,12]$. Это позволяет корректным образом учесть в эффективном гамильтониане ГС интерфейсные поправки, обусловленные изменением зонных параметров материалов на гетерогранице, а также поправки, связанные с наличием короткодействующей части интерфейсного потенциала.

В настоящей работе данный подход используется для расчета энергетического спектра и огибающих волновых функций электронов в туннельно-связанных сферических KT InAs/GaAs, образующих простую кубическую 3D CP. Исследуется зависимость энергии основного состояния электрона в центре зоны Бриллюэна СР $(\mathbf{K}=0)$ от радиуса КТ $R(R \approx 7-27 \AA)$. Расчет проводится в рамках однозонной $\Gamma_{1}$-модели без учета деформационных эффектов и спин-орбитального взаимодействия. Примитивные векторы трансляции прямой $\mathbf{D}_{1,2,3}$ и обратной $\mathbf{G}_{1,2,3} \mathrm{CP}$ определяются соответственно как $\mathbf{D}_{1}=D_{0}(1,0,0), \mathbf{D}_{2}=D_{0}(0,1,0), \mathbf{D}_{3}=D_{0}(0,0,1)$ и $\mathbf{G}_{1}=G_{0}(1,0,0), \mathbf{G}_{2}=G_{0}(0,1,0), \mathbf{G}_{3}=G_{0}(0,0,1)$, где $D_{0}=p a_{0}, G_{0}=2 \pi / D_{0}, a_{0}$ - постоянная решетки InAs, $p=13$. В соответствии с этим объем сверхъячейки $\Omega_{s}=N \Omega_{0}$, где $N=4 p^{3}$ - число элементарных ячеек кристалла в сверхъячейке $(N=8788), \Omega_{0}=a_{0}^{3} / 4-$ объем элементарной ячейки.

Согласно [13], уравнение Шредингера для электрона в СР в k-представлении для простой невырожденной зоны имеет вид

$$
\sum_{j} H\left(\mathbf{k}_{i}, \mathbf{k}_{j} ; \mathbf{K}\right) F\left(\mathbf{k}_{j} ; \mathbf{K}\right)=E(\mathbf{K}) F\left(\mathbf{k}_{i} ; \mathbf{K}\right) .
$$

Здесь $H\left(\mathbf{k}_{i}, \mathbf{k}_{j} ; \mathbf{K}\right) \quad$ - эффективный матричный $k p$-гамильтониан, параметрически зависящий от волнового вектора электрона в зоне Бриллюэна CP $\mathbf{K} ; F\left(\mathbf{k}_{j} ; \mathbf{K}\right)$ - огибающая волновая функция 


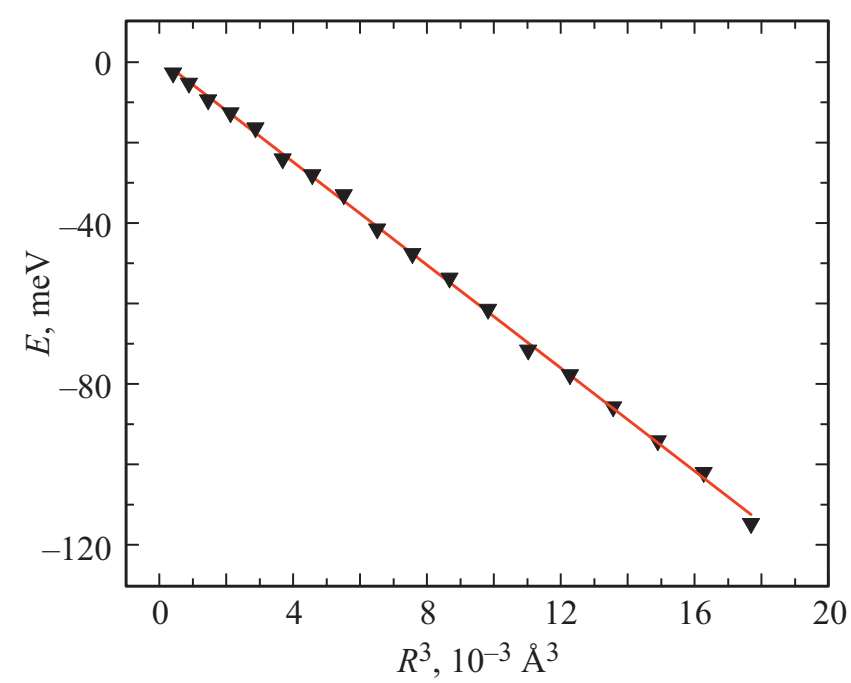

Рис. 1. Зависимость энергии основного состояния электрона $(\mathbf{K}=0)$ от радиуса сферических КТ. Разрыв зоны проводимости $\Delta E=0.55 \mathrm{eV}$. Энергия отсчитывается от дна зоны проводимости GaAs.

электрона $\quad$ в $\mathbf{k}$-представлении; $\mathbf{k}_{i, j} \quad$ - волновые векторы, пробегающие дискретный ряд значений $(i, j=1 \ldots N)$ и равные векторам обратной СР $\mathbf{G}=m_{1} \mathbf{G}_{1}+m_{2} \mathbf{G}_{2}+m_{3} \mathbf{G}_{3} \quad\left(m_{1,2,3}=0, \pm 1, \pm 2 \ldots\right)$, попадающим в зону Бриллюэна кристалла. Без учета поправок от короткодействующей части интерфейсного потенциала эффективный гамильтониан представим в виде [12]:

$$
\begin{aligned}
& H\left(\mathbf{k}_{i}, \mathbf{k}_{j} ; \mathbf{K}\right)=E_{\mathrm{InAs}}^{\Gamma_{1}} \delta_{i j}+\Delta E f\left(\mathbf{k}_{i}+\mathbf{k}_{j}\right) \\
& +\frac{\hbar^{2}\left(\mathbf{k}_{i}+\mathbf{K}\right)^{2}}{2 m_{\mathrm{InAs}}^{*}} \delta_{i j}+\frac{\hbar^{2}}{2}\left(\frac{1}{m_{\mathrm{GaAs}}^{*}}-\frac{1}{m_{\mathrm{InAs}}^{*}}\right) \\
& \times\left(\mathbf{k}_{i}+\mathbf{K}\right) \cdot\left(\mathbf{k}_{j}+\mathbf{K}\right) f\left(\mathbf{k}_{i}-\mathbf{k}_{j}\right) .
\end{aligned}
$$

Здесь в качестве опорного кристалла выбран InAs, $E_{\mathrm{InAs}}^{\Gamma_{1}}-$ энергия, определяющая положение дна зоны проводимости InAs, $\Delta E=E_{\mathrm{GaAs}}^{\Gamma_{1}}-E_{\mathrm{InAs}}^{\Gamma_{1}}-$ разрыв зоны $\Gamma_{1}$ в ГC, $m_{\mathrm{InAs}}^{*}$ и $m_{\mathrm{GaAs}}^{*}$ - эффективные массы электронов в зоне $\Gamma_{1}$ в InAs и GaAs соответственно,

$$
f\left(\mathbf{k}_{i}-\mathbf{k}_{j}\right)=\frac{1}{N} \sum_{l} f\left(\mathbf{a}_{l}\right) e^{-i\left(\mathbf{k}_{i}-\mathbf{k}_{j}\right) \mathbf{a}_{l}}
$$

- фурье-образ характеристической функции $f\left(\mathbf{a}_{l}\right)$, которая принимает значение 1 , если в элементарной ячейке с номером $\mathbf{a}_{l}$ происходит замещение атомов InAs атомами GaAs, и 0, если такого замещения нет. Суммирование в (3) производится по всем векторам прямой решетки $\mathbf{a}_{l}$ (узлам решетки Браве), попадающим в сверхъячейку СР $(l=1 \ldots N)$. Последнее слагаемое в правой части (2) следует рассматривать как поправку к методу эффективной массы, учитывающую изменение эффективной массы электрона при переходе через интерфейс. В континуальном приближении эта поправка приводит к разрыву на интерфейсах первых производных от огибающих волновых функций.

Таким образом, в рассматриваемом нами подходе определение энергетического спектра электрона и его огибающих волновых функций в k-представлении сводится к поиску параметрически зависящих от $\mathbf{K}$ собственных чисел и собственных столбцов матричного гамильтониана (2), размерность которого $N \times N$. Переход к огибающим волновым функциям в а-представлении $F\left(\mathbf{a}_{l} ; \mathbf{K}\right)$ осуществляется посредством обратного фурье-преобразования

$$
F\left(\mathbf{a}_{l} ; \mathbf{K}\right)=\frac{1}{\sqrt{N}} \sum_{i} F\left(\mathbf{k}_{i} ; \mathbf{K}\right) e^{i \mathbf{k}_{i} \mathbf{a}_{l}},
$$

где экспоненциальные функции $(1 / \sqrt{N}) e^{i \mathbf{k}_{i} \mathbf{a}_{l}}$ удовлетворяют следующим условиям ортонормированности и полноты:

$$
\begin{gathered}
\frac{1}{N} \sum_{l} e^{-i\left(\mathbf{k}_{i}-\mathbf{k}_{j}\right) \mathbf{a}_{l}}=\delta_{i j}, \\
\frac{1}{N} \sum_{l} e^{i \mathbf{k}_{l}\left(\mathbf{a}_{i}-\mathbf{a}_{j}\right)}=\delta_{i j} .
\end{gathered}
$$

Огибающая волновая функция в $\mathbf{x}$-представлении определяется следующим соотношением [13]:

$$
F(\mathbf{x} ; \mathbf{K})=\frac{1}{\sqrt{N}} \sum_{i} F\left(\mathbf{k}_{i} ; \mathbf{K}\right) e^{i \mathbf{k}_{i} \mathbf{x}} .
$$

Поскольку область, в которой определены $N$ векторов $\mathbf{k}_{i}$, ограничена зоной Бриллюэна кристалла, это соотношение в отличие от (4) не является обратным преобразованием Фурье.

На рис. 1 и 2 представлены зависимость энергии основного состояния электрона в СР в центре зоны Бриллюэна $(\mathbf{K}=0)$ от радиуса квантовой точки $R$ и распределение электронной плотности $|F(\mathbf{x} ; 0)|^{2}$ в

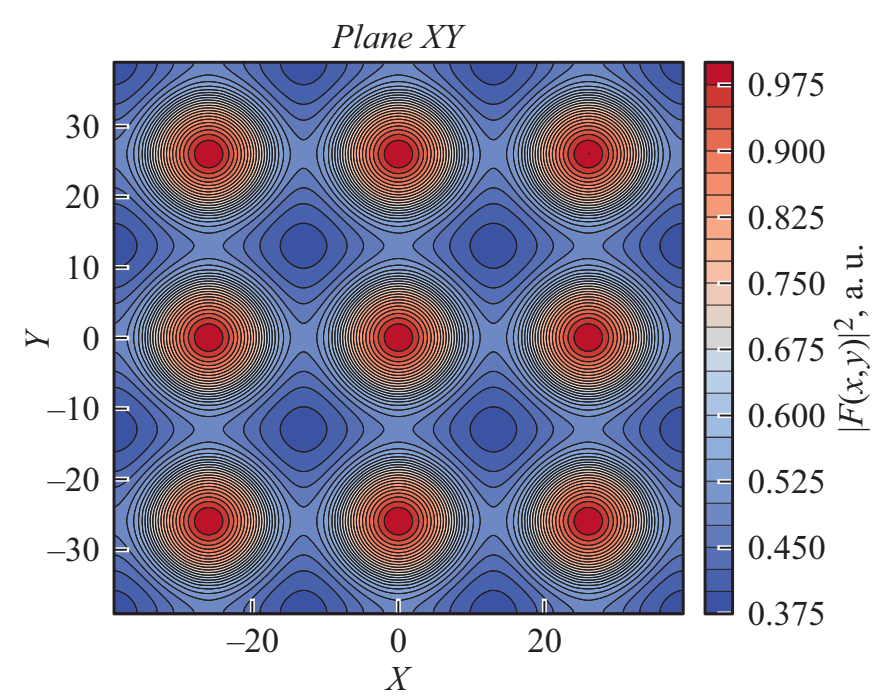

Рис. 2. Распределение электронной плотности, соответствующее основному состоянию электрона $(\mathbf{K}=0)$ в $\mathrm{CP}$. 
кристаллографической плоскости (001). Как следует из рис. 1, энергия связи электрона в области $R<27 \AA$ пропорциональна $R^{3}$.

\section{Финансирование работы}

Работа выполнена при финансовой поддержке Министерства образования и науки РФ (государственное задание № 16.1750.2017/4.6).

\section{Конфликт интересов}

Авторы заявляют, что у них нет конфликта интересов.

\section{Список литературы}

[1] Hammerschmidt T., Kratzer P., Scheffler M. // Phys. Rev. B. 2008. V. 77. P. $235303(1-16)$ DOI: 10.1103/PhysRevB.77.235303

[2] Wang L.W., Kim J., Zunger A. // Phys. Rev. B. 1999. V. 59. P. 5678-5687.

[3] Wang L.W., Zunger A. // Phys. Rev. B. 1999. V. 59. P. 15806 15818.

[4] Williamson A.J., Zunger A. // Phys. Rev. B. 1999. V. 59. P. $15819-15824$.

[5] Williamson A.J., Wang L.W., Zunger A. // Phys. Rev. B. 2000. V. 62. P. $12963-12977$.

[6] Luo J.W., Bester G., Zunger A. // Phys. Rev. B. 2009. V. 79. P. 125329 (1-15). DOI: 10.1103/PhysRevB.79125329

[7] Wang L.W., Zunger A. // Phys. Rev. B. 1996. V. 54. P. $11417-$ 11435.

[8] Fu H., Wang L.W., Zunger A. // Phys. Rev. B. 1998. V. 57. P. 9971-9987.

[9] Stier O., Grundmann M., Bimberg D. // Phys. Rev. B. 1999. V. 59. P. $5688-5701$.

[10] Moskalenko A.S., Berakdar J., Prokofiev A.A., Yassievich I.N. // Phys. Rev. B. 2007. V. 76. P. 085427 (1-9). DOI: 10.1103/PhysRevB.76.085427

[11] Глинский Г.Ф. Полупроводники и полупроводниковые наноструктуры: симметрия и электронные состояния. СПб: Технолит, 2008. 324 c. http://www.twirpx.com/file/1014651/

[12] Глинский Г.Ф., Миронова М.С. // ФТП. 2014. Т. 48. В. 10. С. $1359-1369$.

[13] Глинский Г.Ф. // Письма в ЖТФ. 2018. Т. 44. В. 6. С. 17-24. DOI: 10.21883/PJTF.2018.06.45763.17113 